Research Paper

\title{
Association between Helicobacter Pylori Infection and Ulcerative Colitis-A Case Control Study from China
}

\author{
Xi Jin ${ }^{* \bowtie}$, Yi-peng Chen", Shao-hua Chen, Zun Xiang \\ Department of Gastroenterology, The First Affiliated Hospital, College of Medicine, Zhejiang University, Hangzhou, P.R. China. \\ * These authors contribute equally to this paper as the first author.
}

$\triangle$ Corresponding author: Xi Jin, Digestive Department, The First Affiliated Hospital, College of Medicine, Zhejiang University, No.79 Qingchun Road, Hangzhou, Zhejiang 310003, P.R. China. Fax: 86-571-87236611 Tel: 86-571-87236603 Email: jxfl007@hotmail.com.

(C) Ivyspring International Publisher. This is an open-access article distributed under the terms of the Creative Commons License (http://creativecommons.org/ licenses/by-nc-nd/3.0/). Reproduction is permitted for personal, noncommercial use, provided that the article is in whole, unmodified, and properly cited.

Received: 2013.06.18; Accepted: 2013.08.12; Published: 2013.08.30

\begin{abstract}
Aims To investigate the association between $H$. pylori infection and UC prevalence in China. Materials and Methods Subjects were selected from patients admitted in Department of Gastroenterology for abdominal pain, hematochezia, diarrhea and other Gl symptoms during 2009-20 I2. UC diagnosis was based on both colonoscopy and biopsy. H. pylori detection was based on ${ }^{14} \mathrm{C}$ urea breath test (UBT) and biopsy sample culture. Patients' demographic, anthropometric and serologic data were selected. $H$. pylori infection rate was compared between UC and control groups, followed by a subgroup analysis on the association between $H$. pylori infection and extent and severity degree of UC. Results Totally, I53 and I2I patients were selected and divided into $\mathrm{UC}$ and control groups. There were no significant differences in age, gender, BMI, hypertension and diabetes. However, smoking history was significantly lower while WBC and CRP levels were significantly higher in UC group. The $H$. pylori infection rate in UC group was $30.5 \%$, significantly lower than that of $57.0 \%$ in control group. The $\mathrm{H}$. pylori infection rate in UC of left colon and whole colon were $33,9 \%$ and $24.2 \%$ ( $p<0.05$ between them), both significantly lower than that in control group. In addition, the $H$. pylori infection rates in mild, moderate and severe UC subgroups were $37.8 \%, 32.3 \%$ and $22.2 \%$ ( $p>0.05$ among them), all of which were significantly lower than that in control group. Conclusion We reported a significantly lower $H$. pylori infection rate in UC patients with different extent and severity degree, which provides evidence for bacteria involvement in UC pathogenesis and reminder clinicians to keep cautious in considering $\mathrm{H}$. pylori eradication in UC patients.
\end{abstract}

Key words: ulcerative colitis, helicobacter pylori, urea breath test.

\section{Introduction}

Ulcerative colitis (UC) belongs to a subgroup of inflammatory bowel disease (IBD) and is characterized as chronic inflammation affecting the entire colon (1). Although genetic, immunologic and environmental factors play important roles in UC, the precise etiology is still unclear. Currently, UC has become a global health threat, which is usually common in developed countries such as northern Europe and the USA (2) and with increasing prevalence in many developing countries since $1990(3,4)$. In china, the hos- pital based UC prevalence has been rising by three times over a 10-year period in Hong Kong (5). In addition, a steady increase of UC has also been observed from 1990 to 2003 in Wuhan city (6). The reason for this apparent increase may be due to increased awareness and diagnosis of UC. Besides, improved access to a cleaner environment and the resulting decreased bacterial infection in children may also contribute to this change $(7,8)$. Therefore, the microbial in human gut may play a pivotal role in the pathogene- 
sis of UC.

Helicobacter pylori (H. pylori) is a type of curved or spiral flagellated, Gram- negative microaerophilic bacterium and has been co-existing with human for over 5000 years $(9,10)$. Since discovered in 1984 (11), $H$. pylori has been recognized as the main risk factor for gastritis, peptic ulcer, gastric carcinoma and gastric mucosa- associated lymphoma $(12,13)$. Nowadays, accumulating evidences indicate that $H$. pylori protects human from various diseases with an auto-immune component (14). This effect may be through skewing the host immunologic tone away from inflammatory Th1/Th17 response (15) and increasing T-regulatory cell level (16). Considering the immune regulation capacity of $H$. pylori and the nature of autoimmune related damage in UC, it is theoretically reasonable that $H$. pylori may be involved in the pathogenesis of UC.

Such speculation has been investigated in various studies. According to epidemiology data, UC is more prevalent in developed countries with lower rates of $H$. pylori colonization than in developing countries with higher $H$. pylori infection (17). For instance, a small scale study showed a low H. pylori infection rate in UC patients in China (18). Besides, a steady rise in UC incidence was reported in $H$. pylori endemic regions after successful $H$. pylori eradication (19). In addition, a meta-analysis of 23 studies suggested a protective role of $H$. pylori infection against IBD development (20). Nevertheless, the heterogeneity among studies and the possibility of publication bias impaired the reliability of this meta-analysis while several studies also reported negative association between $H$. pylori and UC $(21,22)$. We previously reported the inverse relationship between $H$. pylori infection and Crohn's disease, another subtype of IBD (23). In this study, we further conducted a large scale case control study to investigate the association between $H$. pylori and UC, especially between different extent and severity degree of UC that is rarely reported.

\section{Methods}

\section{Ethics statement}

The protocol was approved by the institutional review board at Zhejiang University and conducted in accordance with the Declaration of Helsinki. The study design and manuscript preparation were based on guideline from the STROBE statement (24). Written informed consent was collected from all patients.

\section{Patients}

Study subjects were selected from patients who were admitted in Department of Gastroenterology for abdominal pain, hematochezia, diarrhea and other GI symptoms during 2009-2012. Only those who had both $H$. pylori test and colonoscopy were further considered. The $H$. pylori test was based on ${ }^{14} \mathrm{C}$ urea breath test (UBT) and biopsy sample culture in patients taking esophageal gastroscopy. As serum $H$. pylori- IgG test cannot reflect current $H$. pylori infection status, H. pylori-IgG positive patients were reconfirmed with above mentioned methods. The diagnosis of UC was based on colonoscopy manifestation and biopsy, as adopted by the Asia-Pacific consensus (3). Exclusion criteria included previous acid inhibition (proton pump inhibitor or $\mathrm{H}_{2}$ receptor antagonist) or $H$. pylori eradication and 5-aminosalicylic administration. The control group was comprised of patients who underwent the initial screening but were subsequently excluded by negative results for UC and other known GI diseases.

To further investigate the association between UC and H. pylori, we categorized UC patients into different subgroups according to disease severity and extent. In detail, based on ESGE recommendation for image documentation in colonoscopy (25) that includes vascular pattern, erythema, edema, granularity, blood in lumen, erosion, ulcerations and friability, we divided UC into mild, moderate and severe degrees. Generally, mild degree represents reduced vascular pattern, some vulnerability; moderate degree represents absent vascular pattern, vulnerable mucosa, erosions; severe degree represents spontaneous bleeding, ulcerations. Such categorization has a good inter-observer agreement, as reported by Dr Thomas de Lange, et al (26). In addition, the extent of UC was divided into involving the whole colon and left side colon.

\section{Analysis of demographic, anthropometric and serologic data}

Patients' demographic and anthropometric data were retrieved from the medical records on enrollment, including age, gender, smoking history, body mass index (BMI, calculated as weight in kilograms divided by height in meters squared), hypertension (defined as a patient on antihypertensive drug for blood pressure over $140 / 90 \mathrm{mmHg}$ ) and diabetes mellitus (DM, defined as fasting glucose $\geq 7.0$ $\mathrm{mmol} / \mathrm{L}$ or with past history of diagnosed DM). Patients' blood samples were routinely collected and tested for general condition and inflammation, including complete blood cell, $\mathrm{C}$ reaction protein (CRP) and so on.

\section{${ }^{14} \mathrm{C}$ UBT and biopsy sample culture}

${ }^{14} \mathrm{C}$ UBT was first described in 1989 (27) as a rapid diagnostic procedure for $H$. pylori detection, 
mainly based on the ability of $H$. pylori to convert urea to ammonia and carbon dioxide. Nowadays, UBT is recommended in leading society guidelines as a preferred non-invasive choice for $H$. pylori detection before and after treatment (28). Briefly, patients take a tablet of urea labeled with an uncommon isotope of radioactive carbon-14. The isotope labeled carbon dioxide in exhaled breath is measured by scintillation in $30 \mathrm{~min}$. A positive result indicates the existence of $H$. pylori in the stomach. Gastric samples biopsied from gastroscopy were routinely cultured for $H$. pylori detection, according to previously developed method (29).

\section{Statistics}

Data were assessed for normality and log-transformed where appropriate. Quantitative variant were expressed as mean \pm standard deviation (SD) or median with range once nonnormal distribution was found. Student $t$ test or Mann-Whiney U-test was further applied. For qualitative variant, percentages or frequencies were calculated and a chi-square test was used for comparison. SPSS 17.0 (Chicago, IL, USA) was used for statistical analysis and $p<0.05$ was considered statistically significant.

\section{Results}

\section{Characteristic of Study Subjects}

Through careful medical record review, we identified 1031 patients that had been admitted in Department of Gastroenterology for abdominal pain, hematochezia, diarrhea and other GI symptoms over the past three years. Among these patients, 832 received both colonoscopy and $H$. pylori test and were selected for further analysis. Among them, 189 patients were diagnosed as UC by colonoscopy and biopsy confirmation, 152 patients as Crohn's disease, 251 patients as different degrees of haemorrhoids, 34 patients as ischemic colitis, 5 patients as antibiotic associated colitis, 3 patients as radiation enterocolitis and 54 patients as sigmoiditis and proctitis. The remaining 144 patients had intestinal symptoms but with normal appearance in both colonoscopy and biopsy. Among them, 23 patients were further excluded as 11 cases were under anti-acid therapy (8 with proton pump inhibitor and 3 with $\mathrm{H}_{2}$ receptor antagonist) and 12 had previous $H$. pylori eradication therapy.

Among 189 UC patients, 12 were under 5-aminosalicylic drug therapy, 7 were under proton pumper inhibitor therapy for reflux symptom and 13 had H. pylori eradication therapy. Among the rest patients, 4 were further excluded for positive in serum H. pylori -IgG but negative in ${ }^{14} \mathrm{C}$ UBT or biopsy sam- ple culture. Finally, there were 153 and 121 patients selected and divided into UC and control groups. In UC group, 81 and 72 patients had ${ }^{14} \mathrm{C}$ UBT and biopsy sample culture, respectively. In control group, 57 and 64 patients had ${ }^{14} \mathrm{C}$ UBT and biopsy sample culture, respectively. There was no significant difference in $H$. pylori detection method between two groups.

\section{Demographic, anthropometric and serologic data of enrolled patients}

As shown in Table 1, the age and gender distribution were balanced between patients from two groups. BMI in UC group was lower than that in control group but not reached statistical significance. The differences in the rates of hypertension and diabetes between two groups were also not significant. However, compared with UC group, rate of smoking history was approximately twice time higher in control group $(\mathrm{p}<0.01)$, reinforcing the previous hypothesis that smoking was protective for UC. In addition, two inflammation and infection associated markers (CRP and $\mathrm{WBC}$ ) were significantly higher in UC group, reconfirming the involvement of inflammation in disease pathogenesis.

Table I. Demographic, anthropometric and serologic data of enrolled patients

\begin{tabular}{llll}
\hline Group & $\begin{array}{l}\text { UC } \\
(\mathrm{n}=153)\end{array}$ & $\begin{array}{l}\text { Control } \\
(\mathrm{n}=121)\end{array}$ & p value \\
\hline Age(y) & $44.6 \pm 10.7$ & $49.1 \pm 7.5$ & 0.12 \\
Gender(M/F) & $79 / 74$ & $57 / 64$ & 0.07 \\
Smoking history(\%) & 17.6 & 33.9 & $<0.01$ \\
BMI & $24.4 \pm 2.2$ & $25.5 \pm 1.8$ & 0.09 \\
Hypertension(\%) & 15.0 & 17.4 & 0.11 \\
Diabetes & 7.2 & 6.6 & 0.19 \\
CRP(mg/L) & $45.3 \pm 14.5$ & $6.9 \pm 2.0$ & $<0.01$ \\
WBC $\left({ }^{*}\right.$ 10E9/L) & $10.8 \pm 2.2$ & $5.4 \pm 1.6$ & $<0.01$ \\
\hline
\end{tabular}

WBC, white blood cell,

\section{Association between UC and $\boldsymbol{H}$. pylori infection}

Total H. pylori infection rate in UC group was $30.5 \%$, significantly lower than that of $57.0 \%$ in control group. In UC group, the extent of UC was restricted in left colon in 112 patients, mainly involving rectum and sigmoid. The other 41 patients had entire colon injury but there were no toxic megacolon and intrusion ileitis. In subgroup analysis, the H. pylori infection rate in both UC of left colon and entire colon groups were significantly lower than that in control group. Intriguingly, H. pylori infection rate in UC of entire colon group was also significantly lower than that in UC of left colon group (Table 2). 
To further investigate the association between $H$. pylori infection and UC, we divided UC group into three subgroups according severity under colonoscopy appearance as indicated in Methods. Briefly, there were 37, 62 and 54 patients in mild, moderate and severe UC subgroups with $H$. pylori positive rate of $37.8 \%, 32.3 \%, 22.2 \%$, respectively (Table 3). All three subgroups had significantly lower H. pylori infection rate than that in control group. However, though there was a declination tendency of $H$. pylori infection rate from mild to severe UC group, the differences among these three groups were not significant.

Table 2. $H$. pylori infection between different extent of UC and control

\begin{tabular}{lllll}
\hline Group & $\begin{array}{l}\text { H. pylori } \\
\text { positive(n) }\end{array}$ & $\begin{array}{l}\text { H. pylori } \\
\text { negative(n) }\end{array}$ & $\begin{array}{l}\text { H. pylori } \\
\text { positive(\%) }\end{array}$ & $\mathrm{p}^{*}$ \\
\hline UC & 46 & 107 & 30.5 & $<0.01$ \\
UC of left colon & 38 & 74 & 33.9 & $<0.01$ \\
UC of whole colon & 8 & 33 & $24.2^{\#}$ & $<0.01$ \\
Control & 69 & 52 & 57.0 & \\
\hline *, compared with control group; \#, compared with UC of left colon, $\mathrm{p}<0.05$
\end{tabular}

*, compared with control group; \#, compared with UC of left colon, $\mathrm{p}<0.05$

Table 3. $H$. pylori infection between different severity of UC and control

\begin{tabular}{lllll}
\hline Group & $\begin{array}{l}\text { H.pylori } \\
\text { positive(n) }\end{array}$ & $\begin{array}{l}\text { H.pylori } \\
\text { negative(n) }\end{array}$ & $\begin{array}{l}\text { H.pylori } \\
\text { positive }(\%)\end{array}$ & $\mathrm{p}^{*}$ \\
\hline Mild UC & 14 & 23 & 37.8 & $<0.05$ \\
Moderate UC & 20 & 42 & 32.3 & $<0.01$ \\
Severe UC & 12 & 42 & 22.2 & $<0.01$ \\
Control & 69 & 52 & 57.0 & \\
\hline
\end{tabular}

*, compared with control group

\section{Discussion}

UC has been considered as a chronic condition affecting the entire human colon with superficial mucosal layer injury. The pathogenesis of UC is still vague while progress in genetics has improved our understandings (30). For instance, Parkes $\mathrm{M}$ reported the involvement of major histocompatibility complex and epithelial barrier related genes for UC through GWAS study (31) while Louis E reinforced the importance of interleukin-10 in UC development (32). Furthermore, the interplay between genetic predisposition, host immunology and bacteria at the mucosal surface becomes a hot research spot while a meta-analysis on GWAS studies revealed the considerable overlap between susceptibility loci for UC and mycobacterial infection (33). Nevertheless, though various organisms were suggested as pathogens for $\mathrm{UC}$, none of them have been conclusively proved (34).
One possible explanation is that gastrointestinal infection may facilitate the change in bacterial populations to the detriment of the host, which further contributes to UC occurrence. Therefore, finding specific bacteria in UC is of vital importance, not only for disease mechanism exploration, but also for potential antibiotic therapy.

It is well known that $H$. pylori is the causative agent in gastric and duodenal ulceration and its potential role in UC has been intensively investigated. Generally, H. pylori had two main subgroups: gastric helicobacter that preferentially colonized the stomach and enterohepatic helicobacter that mainly infected intestinal or hepatobiliary system (35). Previous studies in immune deficient rodents showed that $H$. pylori was capable to cause IBD like disease by initiating "auto-immune" type reaction $(36,37)$. Moreover, Ram M, et al reported the association between $H$. pylori and IBD (38). Nevertheless, clinical trials investigating the association between $H$. pylori and UC showed confusing results. On one hand, Oliveira AG, et al(21) and Parlak E, et al (22) failed to find any association between $H$. pylori infection and UC prevalence. Besides, H. pylori was not identified in colonic biopsies from UC patients by helicobacter genus-specific PCR assay (39) and nested PCR assay (40). On the other hand, He jin-de, et al found a significantly lower $H$. pylori infection in UC patents than that in patients with chronic gastritis by H. pylori-IgG detection (41), which was further reconfirmed by Halme, et al with the same method (42). However, Pearce et al further indicated that such positive association was irrelevant with antibiotic therapy (43).

To tackle this discordance, we retrospectively investigated the association between $H$. pylori infection and UC in a large case control study of Chinese patients. The initial results showed that $H$. pylori infection was significantly lower than that in control group, providing solid supplementary for previous meta-analysis(20). More importantly, the ${ }^{14} \mathrm{C}$ UBT and biopsy sample culture had higher sensitivity and specificity than serum $H$. pylori-IgG test in several studies, increasing the credibility of these findings. The BMI and hypertension rates were relatively lower in enrolled UC patients, which may be due to malnutrition caused by diarrhea and other GI symptoms. Differing from previous studies, we added subgroup analysis on $H$. pylori infection and UC. As shown in Table 2 and 3, we found significantly lower H. pylori infection in each subgroup of UC patients. More importantly, there seemed a trend that the extent and severity of UC increased with $H$. pylori infection decreasing. This finding further put weight on the positive association between $H$. pylori and UC.

It is theoretically plausible for the protective role 
of $H$. pylori infection in UC. First, H. pylori was found to be involved in various autoimmune diseases, including giant cell arteritis, systemic sclerosis and primary biliary cirrhosis (38). Other studies also reported the association between $H$. pylori and autoimmune bollous diseases (44) and pernicious anemia (45). Since autoimmune reaction is involved in UC pathogenesis, H. pylori may take effect through influencing this process. Second, previous research showed that $H$. pylori was able to influence intestinal immune reaction through triggering $\mathrm{TH} 1$ dominated cell defense, which may further decrease immunity related intestinal injury (46). Finally, H. pylori may induce production of antibacterial peptide to inhibit other bacteria caused inflammatory bowel disease (47).

Several limitations of this study should be acknowledged. First, we did not test $H$. pylori in colon biopsy, which may decrease the disease prevalence rate. Second, it is better to use ${ }^{13} \mathrm{C}$ UBT instead of ${ }^{14} \mathrm{C}$ UBT, since the former has no radiation and much safer for patients (48). Third, the trend of decreased $H$. pylori infection paralleling with increased extent and severity degree of UC should be investigated in a larger clinical trial for statistical significance. Finally, the causative role of $H$. pylori infection in UC pathogenesis cannot be established through case control study and further prospective clinical trial is needed. In summary, we reported a significantly lower $H$. pylori infection in UC patients in a large scale case control study. More meaningfully, subgroup analysis showed the trend of association between decreased $H$. pylori infection and increased extent and severity degree of UC. These results provide evidence for bacteria involvement in UC pathogenesis and reminder clinicians to keep cautious in deciding H. pylori eradication in UC patients.

\section{Competing Interests}

The authors have declared that no competing interest exists.

\section{Funding}

This study is sponsored by the National Natural Science Foundation of China (Project 81000169, 81100277, 81200284), Excellent Young Investigator Foundation of Health Bureau of Zhejiang Province (Project 2010QNA011), Excellent Young Investigator Natural Science Foundation of Zhejiang province (Project R2110159) and Project of Zhejiang Traditional Chinese Medicine Administration Bureau (Project 2010ZA065). The founders had no role in study design, data collection and analysis, decision to publish, or preparation of the manuscript. We confirm we will organize payment should the article be accepted for publication.

\section{References}

1. Danese S, Fiocchi C. Ulcerative colitis. The New England journal of medicine. 2011;365(18):1713-25

2. Loftus EV, Jr. Clinical epidemiology of inflammatory bowel disease: Incidence, prevalence, and environmental influences. Gastroenterology. 2004;126(6):1504-17.

3. Ooi CJ, Fock KM, Makharia GK, et al. The Asia-Pacific consensus on ulcerative colitis. Journal of gastroenterology and hepatology. 2010;25(3):453-68.

4. Sood A, Midha V. Epidemiology of inflammatory bowel disease in Asia. Indian journal of gastroenterology. 2007;26(6):285-9.

5. Lok $\mathrm{KH}$, Hung $\mathrm{HG}, \mathrm{Ng} \mathrm{CH}$, et al. Epidemiology and clinical characteristics of ulcerative colitis in Chinese population: experience from a single center in Hong Kong. Journal of gastroenterology and hepatology. 2008;23(3):406-10.

6. Jiang L, Xia B, Li J, et al. Retrospective survey of 452 patients with inflammatory bowel disease in Wuhan city, central China. Inflammatory bowel diseases. 2006;12(3):212-7.

7. Bloomfield SF, Stanwell-Smith R, Crevel RW, et al. Too clean, or not too clean: the hygiene hypothesis and home hygiene. Clinical and experimental allergy : journal of the British Society for Allergy and Clinical Immunology. 2006;36(4):402-25.

8. Koloski NA, Bret L, Radford-Smith G. Hygiene hypothesis in inflammatory bowel disease: a critical review of the literature. World journal of gastroenterology 2008;14(2):165-73.

9. Linz B, Balloux F, Moodley Y, et al. An African origin for the intimate association between humans and Helicobacter pylori. Nature. 2007;445(7130):915-8.

10. Falush D, Wirth $\mathrm{T}$, Linz $\mathrm{B}$, et al. Traces of human migrations in Helicobacter pylori populations. Science. 2003;299(5612):1582-5.

11. Marshall BJ WJ. Unidentified curved bacilli in the stomach of patients with gastritis and peptic ulceration. Lancet. 1984;1:1311-15.

12. Wotherspoon AC, Ortiz-Hidalgo C, Falzon MR, et al. Helicobacter pylori-associated gastritis and primary B-cell gastric lymphoma. Lancet. 1991;338(8776):1175-6.

13. Parsonnet J, Friedman GD, Vandersteen DP, et al. Helicobacter pylori infection and the risk of gastric carcinoma. The New England journal of medicine. 1991;325(16):1127-31

14. van Amsterdam $\mathrm{K}$, van Vliet $\mathrm{AH}$, Kusters JG, et al. Of microbe and man: determinants of Helicobacter pylori-related diseases. FEMS microbiology reviews. 2006;30(1):131-56.

15. Lundgren A, Suri-Payer E, Enarsson K, et al. Helicobacter pylori-specific CD4+ CD25high regulatory T cells suppress memory T-cell responses to $\mathrm{H}$. pylori in infected individuals. Infection and immunity. 2003;71(4):1755-62.

16. Rad R, Brenner L, Bauer S, et al. CD25+/Foxp3+ T cells regulate gastric inflammation and Helicobacter pylori colonization in vivo. Gastroenterology. 2006;131(2):525-37.

17. Danese S, Sans M, Fiocchi C. Inflammatory bowel disease: the role of environmental factors. Autoimmunity reviews. 2004;3(5):394-400.

18. Li Xiu-qing, Pan Li-juan, Tao Zhi-qiang. correlation study between Helicobacter Pylori and ulcerative colitis. Modern Medical Journal. 2010;38(6):647-48

19. Thia KT, Loftus EV Jr., Sandborn WJ, et al. An update on the epidemiology of inflammatory bowel disease in Asia. The American journal of gastroenterology. 2008;103(12):3167-82.

20. Luther J, Dave M, Higgins PD, et al. Association between Helicobacter pylori infection and inflammatory bowel disease: a meta-analysis and systematic review of the literature. Inflammatory bowel diseases. 2010;16(6):1077-84.

21. Oliveira AG, das Gracas Pimenta Sanna M, Rocha GA, et al. Helicobacter species in the intestinal mucosa of patients with ulcerative colitis. Journal of clinical microbiology. 2004;42(1):384-6.

22. Parlak E, Ulker A, Disibeyaz $S$, et al. There is no significant increase in the incidence of Helicobacter pylori infection in patients with inflammatory bowel disease in Turkey. Journal of clinical gastroenterology. 2001;33(1):87-8.

23. Xiang Z, Chen YP, Ye YF, et al. Helicobacter pylori and Crohn's disease: A retrospective single-center study from China. World journal of gastroenterology. 2013;19(28):4576-81.

24. von Elm E, Altman DG, Egger M, et al. The Strengthening the Reporting of Observational Studies in Epidemiology (STROBE) statement: guidelines for reporting observational studies. Lancet. 2007; 370(9596):1453-7. 
25. Rey JF, Lambert R. ESGE recommendations for quality control in gastrointestinal endoscopy: guidelines for image documentation in upper and lower GI endoscopy. Endoscopy. 2001;33(10):901-3.

26. de Lange $T$, Larsen $S$, Aabakken L. Inter-observer agreement in the assessment of endoscopic findings in ulcerative colitis. BMC gastroenterology. 2004;4:9.

27. Surveyor I, Goodwin CS, Mullan BP, et al. The 14C-urea breath-test for the detection of gastric Campylobacter pylori infection. The Medical journal of Australia. 1989;151(8):435-9.

28. Chey WD, Wong BC. American College of Gastroenterology guideline on the management of Helicobacter pylori infection. The American journal of gastroenterology. 2007;102(8):1808-25.

29. Megraud F, Lehours P. Helicobacter pylori detection and antimicrobial susceptibility testing. Clinical microbiology reviews. 2007;20(2):280-322.

30. Thompson AI, Lees CW. Genetics of ulcerative colitis. Inflammatory bowel diseases. 2011;17(3):831-48.

31. Parkes M. The genetics universe of Crohn's disease and ulcerative colitis. Digestive diseases (Basel, Switzerland). 2012;30 Suppl 1:78-81.

32. Louis E, Libioulle C, Reenaers C, et al. Genetics of ulcerative colitis: the come-back of interleukin 10. Gut. 2009;58(9):1173-6.

33. Jostins L, Ripke S, Weersma RK, et al. Host-microbe interactions have shaped the genetic architecture of inflammatory bowel disease. Nature. 2012;491(7422):119-24.

34. Sasaki M, Klapproth JM. The role of bacteria in the pathogenesis of ulcerative colitis. Journal of signal transduction. 2012;: 7049-53.

35. Hansen R, Thomson JM, Fox JG, et al. Could Helicobacter organisms cause inflammatory bowel disease? FEMS immunology and medical microbiology. 2011;61(1):1-14.

36. Kullberg MC, Ward JM, Gorelick PL, et al. Helicobacter hepaticus triggers colitis in specific-pathogen-free interleukin-10 (IL-10)-deficient mice through an IL-12- and gamma interferon-dependent mechanism. Infection and immunity. 1998;66(11):5157-66.

37. Jergens AE, Wilson-Welder JH, Dorn A, et al. Helicobacter bilis triggers persistent immune reactivity to antigens derived from the commensal bacteria in gnotobiotic C3H/HeN mice. Gut. 2007;56(7):934-40.

38. Ram M, Barzilai O, Shapira Y, et al. Helicobacter pylori serology in autoimmune diseases - fact or fiction? Clinical chemistry and laboratory medicine : CCLM / FESCC. 2013;51(5):1075-82.

39. Bell SJ, Chisholm SA, Owen RJ, et al. Evaluation of Helicobacter species in inflammatory bowel disease. Alimentary pharmacology \& therapeutics. 2003;18(5): 481-6.

40. Grehan M, Danon S, Lee A, et al. Absence of mucosa-associated colonic Helicobacters in an Australian urban population. Journal of clinical microbiology. 2004;42(2):874-6.

41. He Jin-de, Ye hui, Zhang Jiao. Serologic presentation of helicobacter pylori infection in patients with inflammatory bowel disease. Journal of Chinese Physician. 2003;5(6):737-38.

42. Halme $\mathrm{L}$, Rautelin $\mathrm{H}$, Leidenius $\mathrm{M}$, et al. Inverse correlation between Helicobacter pylori infection and inflammatory bowel disease. Journal of clinical pathology. 1996;49(1):65-7.

43. Pearce CB, Duncan HD, Timmis L, et al. Assessment of the prevalence of infection with Helicobacter pylori in patients with inflammatory bowel disease. European journal of gastroenterology \& hepatology. 2000;12(4):439-43.

44. Sagi L, Baum S, Agmon-Levin N, et al. Autoimmune bullous diseases the spectrum of infectious agent antibodies and review of the literature. Autoimmunity reviews. 2011;10(9):527-35.

45. Banka S, Ryan K, Thomson W, et al. Pernicious anemia - genetic insights. Autoimmunity reviews. 2011;10(8):455-9.

46. Tursi A. Onset of Crohn's disease after Helicobacter pylori eradication. Inflammatory bowel diseases. 2006;12(10):1008-9.

47. Wehkamp J, Fellermann K, Herrlinger KR, et al. Mechanisms of disease: defensins in gastrointestinal diseases. Nature clinical practice Gastroenterology \& hepatology. 2005;2(9):406-15.

48. Thomas JE. 13C urea breath test. Gut. 1998;43 Suppl 3:S7-12. 